\title{
RDUS
}

Revue de DROIT UNIVERSITÉ DE SHERBROOKE

Titre : $\quad$ QUELQUES CONSEILS POUR BIEN AMORCER UNE MÉDIATION

Auteur(s): $\quad$ Jean POITRAS

Revue: $\quad$ RDUS, 2001-2002, volume 32, numéro 2

Pages: $\quad 475-485$

ISSN : $\quad 0317-9656$

Éditeur: $\quad$ Université de Sherbrooke. Faculté de droit.

URI : $\quad$ http://hdl.handle.net/11143/12331

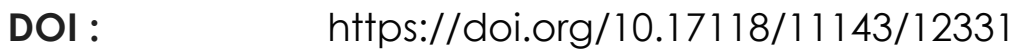


Page vide laissée intentionnellement. 


\begin{abstract}
ARTICLE

\section{QUELQUES CONSEILS POUR BIEN AMORCER UNE MÉDIATION}

\author{
par Jean POITRAS ${ }^{*}$
}

Asseoir les parties à la table de négociation est d'abord et souvent la tâche la plus difficile pour un médiateur. Malheureusement, la documentation vouée à la médiation omet souvent l'amorce du processus. L'objectif de cet article est de mieux faire comprendre les défis à relever pour amener les parties à la table et de proposer des stratégies pour aider à surmonter cet obstacle. L'hypothèse fondamentale sur laquelle repose cet article est qu'une bonne amorce incitera les parties à s'asseoir à la table de négociation. Les parties s'engageront dans une médiation une fois qu'elles auront été convaincues -au cours de l'amorce - qu'il est possible d'en arriver à une entente. L'article présente des stratégies pour atteindre ce but.
\end{abstract}

Getting parties to the negotiating table is the first and often the most difficult task for a mediator. Unfortunately, the initiation phase of the mediation process has often being glossed over in the literature. The purpose of this article is to better understand the challenge of getting the parties to the table and to propose strategies in order to overcome this difficulty. The underlying assumption of this article is that a positive initiation process will encourage parties to come to the negotiating table. Parties engage in mediation when they have come to believe, during this initial phase, that a consensus is possible. The article proposes various strategies to achieve this goal.

* $\quad$ Ph. D., Professeur adjoint au Programme en prévention et en règlement des différends de la Faculté de droit de l'Université de Sherbrooke. 


\section{SOMMAIRE}

Introduction $\ldots \ldots \ldots \ldots \ldots \ldots \ldots \ldots \ldots \ldots \ldots \ldots \ldots \ldots \ldots$

I. Obstacles à asseoir les parties à la table $\ldots \ldots \ldots \ldots 77$

II. Assurer le succès de son amorce . . . . . . . . . . . . . 480

A. Mettre l'accent sur des questions moins controversées .. 480

B. Évaluer la nécessité de résoudre les conflits par voie de collaboration ..................... 481

C. Faire participer les parties à la conception du processus

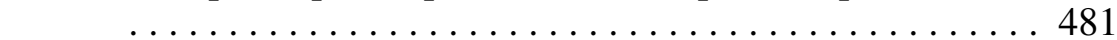

D. Mettre sur pied un forum qui encourage le dialogue ... 482

E. Organiser des rencontres pour promouvoir la connaissance .................... 482

F. Bâtir la confiance progressivement .......... 483

G. Évaluer les avantages de la participation .......... 483

H. Évaluer la valeur d'une relation de travail . . . . . . . . 484

I. Éduquer les parties en matière de collaboration . . . . . . 484

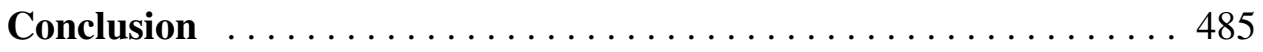

Tableau 1 : Obstacles à asseoir les parties à la table . . . . . . . . 478

Tableau 2 : Stratégies pour réussir son amorce $\ldots \ldots \ldots \ldots 481$ 


\section{Introduction}

Comment fait-on pour faire participer les parties à une médiation? Cette simple question soulève un principe fondamental sous-jacent : la médiation n'est pas possible à moins que les parties ne s'engagent à s'asseoir à la table. Préparer le terrain pour une séance de médiation constitue ainsi une partie intégrante du processus de médiation, ce qu'on pourrait nommer l'«amorce». Puisque les médiateurs doivent continuellement convaincre les parties de s'asseoir à la table, le besoin de mieux comprendre cette amorce devient manifeste.

Malheureusement, en passant en revue la documentation vouée à la médiation, on se rend compte que l'amorce du processus de médiation n'y est peu abordée. Les chercheurs se sont traditionnellement penchés sur la définition et la compréhension des variables en cause dans une négociation une fois que le processus a été mis en branle. De la même façon, l'analyse menée dans les études de cas vise habituellement à prescrire la meilleure démarche possible pour gérer le processus de médiation, mais n'explique que sommairement comment l'entreprendre. Ainsi, une phase critique du processus de médiation est souvent négligée.

Le présent article a pour but de proposer des conseils pour réussir à convaincre les parties en cause de s'asseoir à la table de négociation. Il se divise en deux parties. D'abord, on y explore quelques raisons pouvant justifier la réticence des parties à participer à un processus de médiation. Ensuite, on y décrit des stratégies pouvant servir à encourager un environnement propice à l'initiation d'efforts pour en arriver à des consensus. L'objectif global de cet article vise à guider les médiateurs tout le long de l'amorce et de leur donner les outils qui leur permettront de convaincre les parties plus efficacement de s'asseoir à la table.

\section{Obstacles à asseoir les parties à la table}

Plusieurs raisons peuvent expliquer la réticence de certaines parties à participer à une médiation. Ces raisons ont été regroupées sous la forme de quatre facteurs pouvant constituer des obstacles à asseoir les parties à la table, notamment : 1 . le caractère nouveau de la médiation, 2 . le manque de mesures incitatives pour en arriver à un compromis, 3. l'incertitude entourant l'aboutissement et 4 . la crainte de la négociation. Ces quatre facteurs sont résumés dans le tableau 1 ci-dessous. 
Le premier facteur négatif touche le caractère nouveau du processus de médiation. Le fait que les parties ne connaissent pas les méthodes de médiation peut les rendre méfiantes ou encore elles peuvent tout simplement ne pas être sensibilisées aux avantages potentiels d'en arriver à un consensus. La résistance des parties peut s'avérer encore plus fondamentale dans le cas où leur culture ne préconise tout simplement pas les compromis. Les montants investis dans d'autres processus de résolution de conflits traditionnels, tels que les procédures judiciaires ou les pressions politiques, peuvent également contribuer à rendre de nouveaux processus tels que la médiation, moins attrayants. Aux yeux de certains, la médiation peut sembler ajouter une procédure à un système administratif déjà encombrant. Pour d'autres, la médiation ne jouit pas du statut juridique officiel que possèdent d'autres procédures. Enfin, il est possible que les parties veuillent tout simplement conserver leurs moyens traditionnels de résolution de conflits.

\section{Tableau 1 : Obstacles à asseoir les parties à la table}

Caractère nouveau de la médiation
- $\quad$ La médiation n'est pas ancrée dans la culture des parties
- $\quad$ Investissement dans d'autres procédures (ex. : juridiques ou politiques)
- $\quad$ Méconnaissance du processus de médiation

Manque de motivation à trouver un compromis

- Désir de tout gagner

- $\quad$ Succès antérieur dans le système judiciaire

- $\quad$ Statu quo satisfaisant

- Réticence à abandonner un avantage juridique ou réglementaire

Incertitude entourant le processus

- $\quad$ Crainte de s'engager sur une période de temps

- $\quad$ Caractère possiblement inconcluant des résultats

Crainte de devoir négocier

- Crainte de se faire exploiter

- $\quad$ Crainte de perdre le contrôle sur l'aboutissement

- $\quad$ Méfiance entre les parties

- $\quad$ Réticence à se placer sur un pied d'égalité avec d'autres 
Un deuxième facteur négatif est le manque de motivation pour en arriver à un compromis. À cet égard, un statu quo satisfaisant peut constituer un facteur de dissuasion efficace. Il est possible que des parties qui se satisfont de leur situation actuelle ne veuillent pas la mettre en péril. Par exemple, une partie peut refuser d'abandonner un avantage juridique ou réglementaire. De plus, les parties bénéficiant de l'avantage dans une situation donnée ne voudront peutêtre pas céder dans ce qu'elles considèrent une «cause gagnante». De la même façon, des procédures judiciaires et des stratégies politiques ayant déjà porté fruit rendront les parties réticentes à conclure ce qu'elles jugent être un compromis non nécessaire. Enfin, certaines parties préconiseront peut-être une alternative qu'elles considèrent préférable à une procédure fastidieuse pour en arriver à un compromis. Cela est notamment le cas lorsqu'une option de rechange irréfutable se présente.

Un troisième facteur pouvant nuire à la participation des parties au processus de médiation est l'incertitude quant à l'aboutissement. Certaines parties hésiteront à consacrer temps et ressources à un processus qui ne leur garantit pas un résultat concluant. On craint, entre autres, que cette nouvelle approche ne soit pas axée sur les objectifs, mais plutôt sur la compréhension. Autrement dit, l'aboutissement n'est pas nécessairement une résolution, mais plutôt une compréhension. On court donc des risques en participant, car il est possible que les parties ne réussissent jamais à s'entendre. Par ailleurs, un consensus ne garantit pas à coup sûr la mise en œuvre des décisions. En fait, certaines parties préféreront une décision obligatoire rendue par les tribunaux à une entente négociée moins officielle, même dans le cas où l'entente négociée est plus avantageuse que la décision du tribunal. Cela peut s'appliquer surtout à des parties qui cherchent à faire valider un règlement ou à créer un précédent jurisprudentiel.

Le quatrième et dernier facteur abordé est la crainte de devoir entreprendre des négociations avec d'autres parties. Dans ce cas, la crainte est associée avec la nouveauté des opposants plutôt qu'avec la nouveauté du processus lui-même. Il est possible que les parties hésitent à entamer des négociations par crainte de se faire exploiter. La méfiance entre les parties peut également les pousser à douter de la possibilité de conclure une entente, à un point tel qu'elles croient impossible toute négociation. Enfin, la réticence de certaines parties à se mettre sur un pied d'égalité avec d'autres peut faire en sorte qu'elles refusent de s'asseoir à la même table que des parties indésirables. 


\section{Assurer le succès de son amorce}

Pour convaincre les parties à s'engager dans un processus de médiation, il est essentiel de s'assurer de l'efficacité de son amorce. Puisque la médiation se définit comme un processus volontaire, aucune partie ne peut être forcée à s'asseoir à la table. En conséquence, la stratégie générale consiste à créer des conditions propices à stimuler l'intérêt des parties envers les avantages potentiels du processus. En mettant en place une amorce efficace, on réussit à convaincre les parties à la médiation de l'attrait de s'asseoir à la table. En adoptant les stratégies décrites ci-dessous ${ }^{1}$, les médiateurs ont plus de chances de réussir à faire participer les parties pleinement et activement au processus.

\section{A. Mettre l'accent sur des questions moins controversées}

En règle générale, il est habituellement plus facile d'entreprendre une médiation sur la base de problèmes moins sujets à controverse qu'en prenant pour point de départ des problèmes résultant de différends sur le plan des valeurs ou des principes ${ }^{2}$. Cela ne laisse aucunement entendre qu' on ne doit pas tenir compte des aspects plus controversés d'un problème; il s'agit plutôt d'entreprendre toute discussion avec des questions moins controversées dans la mesure du possible. Le problème devra possiblement être redéfini si l'accent est mis sur des questions moins controversées. Par exemple, au lieu de discuter du bien-fondé d'un projet, les parties voudront peut-être définir les critères d'acceptabilité utilisés pour juger un projet quelconque. Une telle définition favoriserait la résolution des problèmes. Toutefois, il peut s'avérer impossible de définir certaines questions controversées en des termes moins controversés. Outre de telles situations, il est important de définir le problème dans un cadre de résolution de problèmes et d'aborder les questions moins controversées en premier, autant qu'il est possible, tout en proposant un programme de négociation.

1. Voir le tableau 2 «Stratégie pour réussir son amorce» de la sous-section A. «Mettre l'accent sur des questions moins controversées», ci-dessous.

2. D.J. Amy, The Politics of Environmental Mediation, New York, Columbia University Press, 1987 à la p. 177. 


\section{Tableau 2 : Stratégies pour réussir son amorce}

1. Mettre l'accent sur les questions moins controversées dans la mesure du possible

2. Évaluer la nécessité de résoudre les conflits par voie de collaboration

3. Faire participer les parties à la conception du processus

4. Mettre sur pied un forum qui encourage le dialogue

5. Organiser des rencontres pour promouvoir la connaissance

6. Bâtir la confiance progressivement

7. Évaluer les avantages de la participation

8. Évaluer la valeur d'une relation de travail conjointement avec les parties

9. Éduquer les parties en matière de collaboration

\section{B. Évaluer la nécessité de résoudre les conflits par voie de collaboration}

En évaluant dans quelle mesure une question doit être résolue, il est possible d'évaluer l'élan initial pour entreprendre une médiation ${ }^{3}$. Par exemple, dans le cas où le statu quo est jugé inacceptable (les coûts associés avec la nonrésolution d'un litige sont faramineux), les parties seront davantage prêtes à collaborer. De la même façon, la menace d'une poursuite si le problème n'est pas résolu peut également exercer une pression sur les parties à régler leurs différends. Il est important de bien différencier la nécessité de résoudre un problème de la nécessité de collaborer. Certaines questions peuvent être importantes sans qu'une collaboration ne soit requise pour les résoudre. Dans d'autres cas, la collaboration est essentielle. En évaluant dans quelle mesure une collaboration est requise, il devient possible d'évaluer l'élan initial pour entreprendre une médiation. Conscientiser les parties à la nécessité et aux avantages d'une collaboration peut s'avérer une des tâches les plus cruciales dans l'entreprise d'une médiation.

\section{Faire participer les parties à la conception du processus}

La méthode décisionnelle utilisée influencera souvent la décision des parties de participer ou non à la médiation. Les parties doivent croire en l'équité du processus de médiation avant d'être en mesure de s'y engager. Lorsque les

3. G.W. Cormick, Strategic Issues in Structuring Multi-Party Public Policy Negotiations (1985) 5:2 Negotiation J. 125 à la p. 126. 
parties sont invitées à concevoir elles-mêmes le processus, elles auront davantage tendance à le considérer équitable que s'il est proposé ou imposé par un tiers ou une seule partie ${ }^{4}$. Parmi les décisions à prendre en concevant le processus, notons l'établissement de l'heure, de la fréquence et de la durée des rencontres, le choix de l'emplacement et la préparation d'un ordre du jour. Les médiateurs qui consultent les parties sur le processus à intervalles réguliers ont plus de chances de les convaincre de l'équité du processus.

\section{Mettre sur pied un forum qui encourage le dialogue}

Un des principaux attraits de la médiation est la possibilité qu'elle offre d'entreprendre des discussions informelles qui permettent aux participants de voir au-delà des gesticulations et des tribunes d'intérêt commun ${ }^{5}$. À cet égard, l'amorce peut également s'avérer le moment propice d'établir les règles de base du processus de sorte à limiter le recours à la gesticulation. Il ne faut pas négliger l'importance de telles règles de base dans la promotion d'un forum de discussion sécuritaire et ouvert. Une médiation peut échouer si les rencontres en personne ne sont pas gérées correctement ${ }^{6}$. Les règles de base définissent habituellement les normes comportementales que les participants sont tenus de respecter. Par exemple, un forum communautaire peut reposer sur deux règles de base : «Vous avez droit à votre opinion, et votre voisin a droit au sien». Certaines parties seront très motivées si elles constatent des différences avec les anciennes façons de faire.

\section{E. Organiser des rencontres pour promouvoir la connaissance}

Il est tout à fait logique de croire qu'il soit plus facile d'encourager la collaboration entre des personnes qui se connaissent qu'entre des étrangers. Ce principe s'applique tout autant à l'amorce d'une médiation. Lorsque les parties ont l'occasion de se rencontrer au préalable, elles ont plus de chances de mieux comprendre leurs intérêts respectifs et de bâtir des relations personnelles ${ }^{7}$. Cette idée, pourtant toute simple, peut servir à définir une étape importante dans

4. W.L. Ury, J.M. Brett et S.B. Goldberg, Getting Disputes Resolved: Designing Systems to Cut the Costs of Conflict, San Francisco (Cal.), Jossey-Bass aux pp. 69-70.

$5 . \quad$ L. Susskind et J. Cruikshank, Breaking the Impasse: Consensual Approaches to Resolving Public Disputes, New York, Basic Books, 1987 à la p. 77.

6. D.A. Straus, «Managing Meetings to Build Consensus» dans L. Susskind, S. Mckearnan et J. Thomas-Larmer, dir., A Comprehensive Guide to Reaching Agreement, Thousand Oaks (Cal.), Sage, 2000 à la p. 288.

7. F.J. Long et M.B. Arnold, The Power of Environmental Mediations, Forth Worth (Tex.), Dryden, 1995 à la p. 135. 
l'entreprise d'une médiation. Il peut s'avérer d'une importance cruciale de s'assurer que les parties se connaissent un peu lorsque vient le moment d'aborder des questions controversées. À cet égard, de petites conversations tenues au début d'une médiation constituent un important composant du processus. Ces conversations ne doivent toutefois pas porter sur les mérites d'entreprendre une médiation, car cela irait à l'encontre de l'objectif qui est d'apprendre à se connaître dans un contexte libre de toute confrontation. Ces conversations initiales doivent plutôt favoriser l'échange d'information tels que les champs d'intérêt, l'expertise et l'expérience.

\section{F. Bâtir la confiance progressivement}

Avant qu'une partie n'accepte de s'engager dans un effort de collaboration, elle doit être convaincue que les autres parties en cause collaboreront dans la même mesure. En conséquence, il faut pouvoir compter sur un minimum de confiance. Lorsqu'un manque de confiance, voire une méfiance, existe entre les parties, la collaboration peut sembler impossible et la médiation, une perte de temps. Dans de telles conditions, la médiation entreprise sera caractérisée par une atmosphère chargée de soupçons et sera probablement vouée à l'échec ${ }^{8}$. La stratégie générale consiste à proposer des activités de collaboration modérées avant de s'engager dans une médiation en bonne et due forme. De telles activités peuvent inclure l'échange de lettres entre les parties faisant état de leur intention de collaborer ainsi que l'échange de renseignements. Il est important de ne pas sous-évaluer l'importance de tels gestes peu coûteux pour bâtir ou rebâtir la confiance entre les parties. Ces activités permettent habituellement aux parties d'évaluer leurs possibilités de travailler ensemble.

\section{G. Évaluer les avantages de la participation}

Il ne sera pas nécessairement toujours possible de trouver un objectif commun dans le cadre d'une médiation. Dans d'autres cas, bien qu'il y ait un objectif commun, ce ne sera peut-être pas suffisant pour motiver les parties. C'est pour cette raison que la deuxième technique proposée consiste à établir les avantages pour chacune. Plus chaque partie bénéficiera d'une médiation, plus elle aura tendance à y participer. Même dans le cas où il n'existe aucun avantage direct ou indirect d'y participer, le fait de s'exclure du processus peut comporter

8. M. Deutsch, «Cooperation and Competition» dans M. Deutsch et P. Coleman, Handbook of Conflict Resolution: Theory and Practice, San Francisco (Cal.), Jossey-Bass, 2000 aux pp. 28-30. 
des risques. Ces risques potentiels peuvent parfois motiver la participation au processus. Une stratégie d'évaluation des risques d'une non-participation consiste à évaluer les options de rechange à une entente négociée. Parfois, les parties n'ont pas évalué, ou surévaluent, ce qu'il leur est possible d'accomplir sans collaboration. À cet égard, il peut s'avérer utile de rendre la décision de participer plus réaliste. Il est important que les médiateurs s'engagent dans une conversation honnête avec les parties, laquelle portera sur ce qu'elles comptent accomplir par l'entremise du processus de médiation.

\section{H. Évaluer la valeur d'une relation de travail}

En plus de permettre de trouver des solutions aux problèmes ou de rédiger un plan d'action future, la médiation offre l'important avantage de bâtir des relations au cours du processus. Ici, la technique proposée consiste à évaluer la valeur d'une relation de travail dans le cadre d'une collaboration. Les avantages peuvent être considérables, surtout lorsqu'une interaction future est prévisible 9 . Par exemple, les organismes gouvernementaux s'intéressent habituellement à établir des relations de travail avec des groupes d'intérêt et des représentants commerciaux, car ils sont habituellement appelés à interagir avec ces parties régulièrement. Évaluer les possibilités d'interactions futures entre des parties peut constituer une étape importante dans l'entreprise d'une médiation.

\section{I. Éduquer les parties en matière de collaboration}

La dernière technique consiste à éduquer les parties sur le processus de médiation de sorte à ce qu'elles se familiarisent avec la situation. Bien que le fait que les parties ne connaissent rien de la médiation ne constitue habituellement pas un obstacle insurmontable à l'amorce d'une médiation, il est important de ne pas négliger l'importance d'éduquer les parties sur les possibilités de collaboration ${ }^{10}$. Par exemple, une meilleure compréhension du processus de médiation peut aider les parties à se rendre compte que le médiateur ne trouvera pas la solution à leur place. De plus, en éduquant les parties, elles se trouvent toutes sur le même plan dans le processus, et l'équilibre informationnel est ainsi rétabli. Une stratégie pour éduquer et motiver les parties consiste à leur présenter des causes semblables ayant abouti pour ainsi nourrir leur espoir dans le processus.

9. J.M. Zubeck et al., «Disputant and Mediator Behaviors Affecting Short Term Success in Mediation» (1992) 36:3 J. Conflict Resolution 546 à la p. 550.

10. L.S. Carpenter, «Choosing Appropriate Mediation Techniques and Strategies» dans L. Susskind, S. Mckearnan et J. Thomas-Larmer, supra note 6 à la p. 62. 


\section{Conclusion}

La prémisse de cet article est qu'une bonne amorce incitera les parties à s'asseoir à la table de négociation. Tandis que la première partie de l'article faisait état des quatre facteurs qui expliquent les réticences de certaines parties à participer à une médiation, la deuxième partie proposait six stratégies pour assurer l'aboutissement du processus préparatoire. En combinant ces idées et ces recommandations, on obtient de saines stratégies pour amorcer un processus de médiation. Le recours aux stratégies proposées devrait aider à créer un contexte propice à diriger les parties vers la table. Ces stratégies sont conçues pour aider les parties à se rendre compte que le processus de médiation est équitable et ne constitue pas une menace, qu'il est possible de travailler avec l'autre partie et que la participation offre des avantages. Dans un tel contexte, et selon notre expérience, les parties accepteront de s'asseoir à la table de négociation. Il importe toutefois de se rappeler qu'il ne s'agit que de la première étape du processus de médiation. Conclure une entente est une toute autre histoire. 